\title{
Geranium trujillense Aedo (Geraniaceae), una nueva especie del Perú
}

\author{
Carlos Aedo
}

\begin{abstract}
AEDO, C. (2011). Geranium trujillense Aedo (Geraniaceae), a new species from Peru. Candollea 66: 435-439. In Spanish, English and French abstracts. A new species, Geranium trujillense Aedo (Geraniaceae), from North Peru is described and illustrated. A morphological comparison to related Geranium digitatum $\mathrm{R}$. Knuth is provided. The key for sericeous-leaved species of Geranium sect. Neoandina Aedo is updated.
\end{abstract}

\section{Key-words}

GERANIACEAE - Geranium - Peru - Taxonomy

\section{Résumé}

AEDO, C. (2011). Geranium trujillense Aedo (Geraniaceae), une nouvelle espèce du Pérou. Candollea 66: 435-439. En espagnol, résumés anglais et français.

La nouvelle espèce Geranium trujillense Aedo (Geraniaceae) du nord du Pérou est décrite et illustrée. Ses affinités morphologiques avec l'espèce la plus proche Geranium digitatum R. Knuth sont discutées. Une clé actualisée pour les espèces à feuilles soyeuses du genre Geranium sect. Neoandina Aedo est fournie. 
Geranium L. es un grupo subcosmopolita de alrededor de 350 especies. El género fue monografiado por KNUTH (1912) en un trabajo que sigue siendo hoy en día esencial en la sistemática de Geranium. MACBRIDE (1949) publicó una revisión preliminar de las especies del Perú. En otros países cercanos se han publicado catálogos o floras locales pero no hay disponible ninguna revisión completa y actualizada para el género en Sudamérica.

Con el propósito de preparar una nueva monografía de Geranium se han revisado algunas secciones sudamericanas (AEDO, 2001; AEDO \& al., 2002, 2003, 2005), entre las que se encuentra la sect. Neoandina Aedo. Esta sección está formada por plantas acaules generalmente en forma de almohadilla, adaptadas a las severas condiciones ambientales de las zonas altas de los Andes. Se caracteriza también por sus címulas unifloras, que nacen directamente de los rizomas y por la falta de indumento glandulífero. En la inicial revisión del grupo (AEDO \& al., 2002) se reconocieron 22 especies y en sucesivas publicaciones se describieron 2 más (AEDO, 2004, 2007). En el presente artículo se describe otra especie de esta sección procedente de los trabajos de exploración de la zona septentrional del Perú.

\section{Geranium trujillense Aedo, spec. nova (fig. 1)}

Typus: Perú. La Libertad: de Chugay a Molino Viejo, 747'S 7746'W, 28.VI.2009, Aedo \& Zapata 16682 (holo-: MA [788262]; iso-: USM).

A simili specie Geranium digitatum R. Knuth differt nova nostra foliorum laminis profundius divisis ac petiolis indumento antrorso praeditis fructuumque rostribus apicali constrictione carentibus.

Hierba perenne de 4,5-6,9 $\mathrm{cm}$ de altura, escapiforme. Rizoma de 7,7-13,3 mm de diámetro, vertical. Hojas en roseta persistente; lámina de 1,2-1,7 ×0,6-1,2 cm, cuneada, digitada, con los segmentos laterales hacia arriba (relación longitud del seno principal/ longitud del segmento central $=0,53-0,70$ ), serícea en ambas caras; segmentos 5, linear-lanceolados, de 0,8-1,3(-1,5) mm de anchura en la base, enteros o con 3 lóbulos hacia el ápice (relación longitud del seno secundario / longitud del segmento central $=0,07-0,12$ ); pecíolos de hasta $4 \mathrm{~cm}$ de longitud, seríceos, con pelos tectores de 0,3-0,6 mm, antrorsos, adpresos; estípulas 8,9-10 × 1-1,6 mm, lanceoladas, con pelos tectores sobre la cara abaxial y margen, glabras por la cara adaxial. Címulas unifloras, solitarias, que nacen directamente del rizoma, sin pedúnculos ni bractéolas; pedicelos de 10-34 mm, seríceos, con pelos tectores de 0,3-0,8 mm, antrorsos, subadpresos. Sépalos de 6,2-7,3 × 1,4-2,2 mm, no acrescentes, con un mucrón de 0,6-1,2 mm, seríceos, con pelos tectores de 0,4-1,5 mm, erecto-patentes. Pétalos desconocidos. Filamentos estaminales de 2,9-4,4 mm, lanceolados, con pelos tectores $0,2-0,6 \mathrm{~mm}$ sobre la cara abaxial y margen; anteras desconocidas. Nectarios 5, glabros. Gineceo desconocido.
Fruto de 10,2-14,3 mm, erecto; mericarpos de 2,2-3,1 $\times 0,9$ $1,7 \mathrm{~mm}$, con pelos tectores de $0,1-0,4 \mathrm{~mm}$, antrorsos, \pm adpresos; rostro de 5,1-7,9 mm, sin ápice estrechado, seríceo, con pelos tectores de 0,1-0,3 mm, erecto-patentes; restos estigmáticos de 1,1-2,4 mm, con 5 lóbulos pelosos. Semillas de 1,9$2,1 \times 1,2-1,4 \mathrm{~mm}$, reticuladas.

Distribución y hábitat. - Norte del Perú (fig. 2), en formaciones de herbáceas y pequeños sufrútices denominadas "jalca", entre 3830-4000 m.

Etimología. - Especie dedicada a la ciudad peruana de Trujillo capital del departamento de La Libertad, donde fue descubierta la especie en el herbario de la Universidad Antenor Orego (HAO).

Fenología. - Florece en mayo y fructifica en junio.

Parátipos. - Perú. La Libertad: Pataz, Piedra Grande, La Paccha-Chilia, $8^{\circ} 7,2^{\prime}$ 'S 77²5,7’W, 9.V.2003, Sagástegui \& al. 17337 (HAO!); de Chugay a Molino Viejo, desvío a Huagil, 7²7,4'S 77²46,6’W, 4.V.2003, Sagástegui \& al. 17208 (HAO!).

Discusión. - Geranium trujillense es en su aspecto general similar a G. digitatum R. Knuth. Ambas especies comparten las hojas digitadas, tan poco frecuentes en el género, cubiertas de un indumento seríceo por ambas caras. No obstante, en G. trujillense los segmentos foliares son más estrechos y más profundamente divididos. Se ha apreciado alguna variación en la forma del ápice del segmento central que en algunas hojas es entero y en otras con 1-2 cortos lóbulos laterales. Geranium trujillense tiene pelos antrorsos en los pecíolos y generalmente también en los pedicelos mientras que en G. digitatum el indumento de pecíolos y pedicelos es retrorso, a veces patente. Además $G$. trujillense tiene estípulas \pm pelosas (no seríceas como en $G$. digitatum), sépalos con el mucrón más largo, filamentos estaminales más cortos, nectarios glabros, y frutos más cortos en los que el rostrum termina abruptamente en los restos estigmáticos (sin el estrechamiento apical que se observa en $G$. digitatum). Las localidades más septentrionales de G. digitatum distan unos $300 \mathrm{~km}$ de las de G. trujillense.

Geranium trujillense fue inicialmente descubierto en el herbario HAO, donde se conservaban dos colecciones: Sagástegui \& al. 17208 y Sagástegui \& al. 17337. Una visita a la localidad de la primera permitió obtener material fructificado que es el que se describe. Lamentablemente el herbario HAO fue destruido por un incendio en junio del $2010 \mathrm{y}$ los mencionados especímenes (algunos con flor) se perdieron. Por el momento el único material disponible carece de pétalos, anteras y gineceo por lo que los datos referidos a estos caracteres se omiten en la descripción.

Es difícil hacer una estimación del estado de conservación de esta especie a la vista de los escasos datos de los que se dispone por el momento. Se conocen dos localidades separadas 


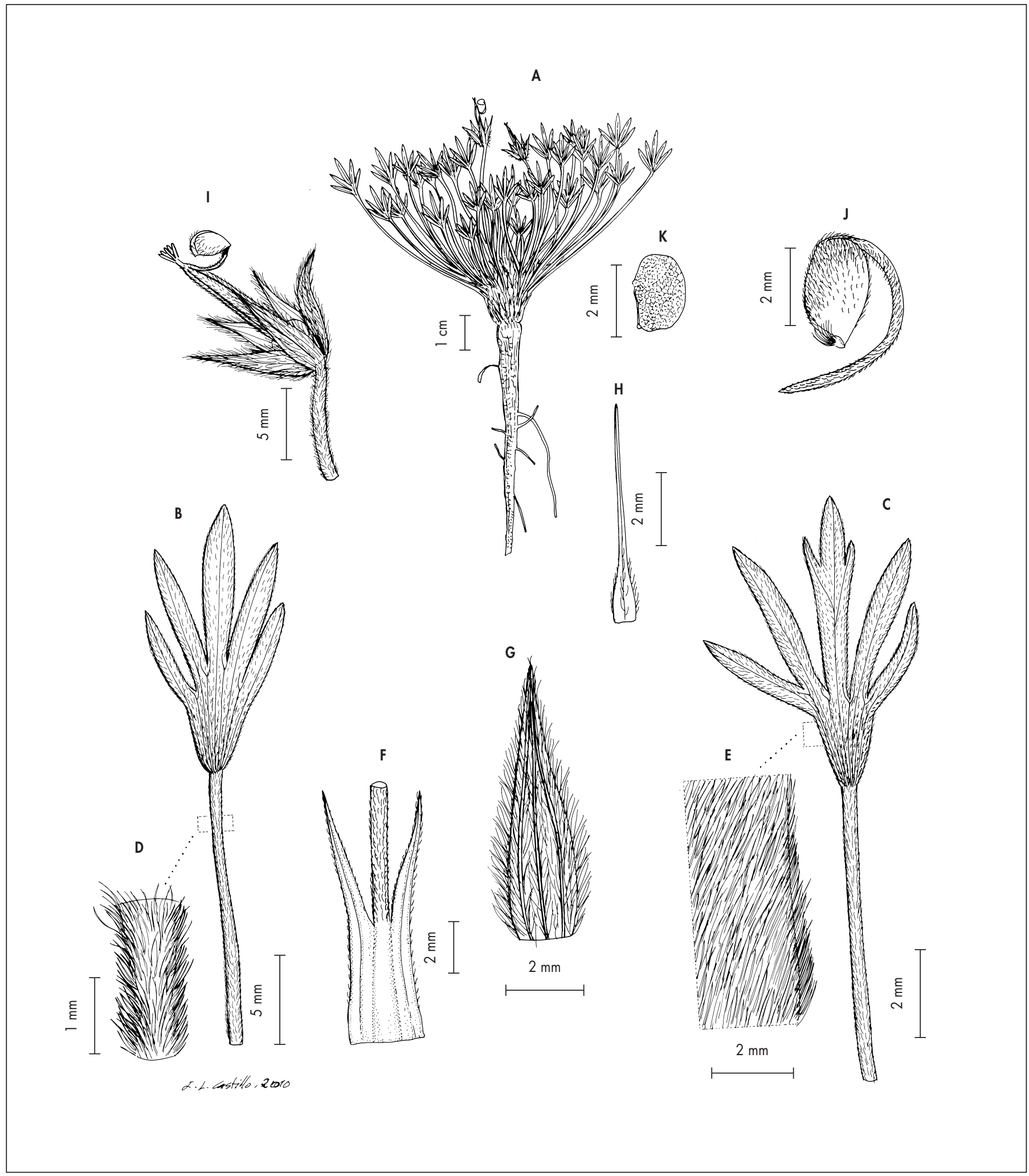

Fig. 1. - Geranium truillense Aedo. A. Habito; B-C. Hojas; D. Indumento del pecíolo; E. Indumento de la lámina foliar; F. Estípulas; G. Sépalo; H. Filamento estaminal ; I. Fruto; J. Mericarpo; K. Semilla.

[Aedo \& Zapata 16682, MA] [Dibujo J. L. Castillo] 


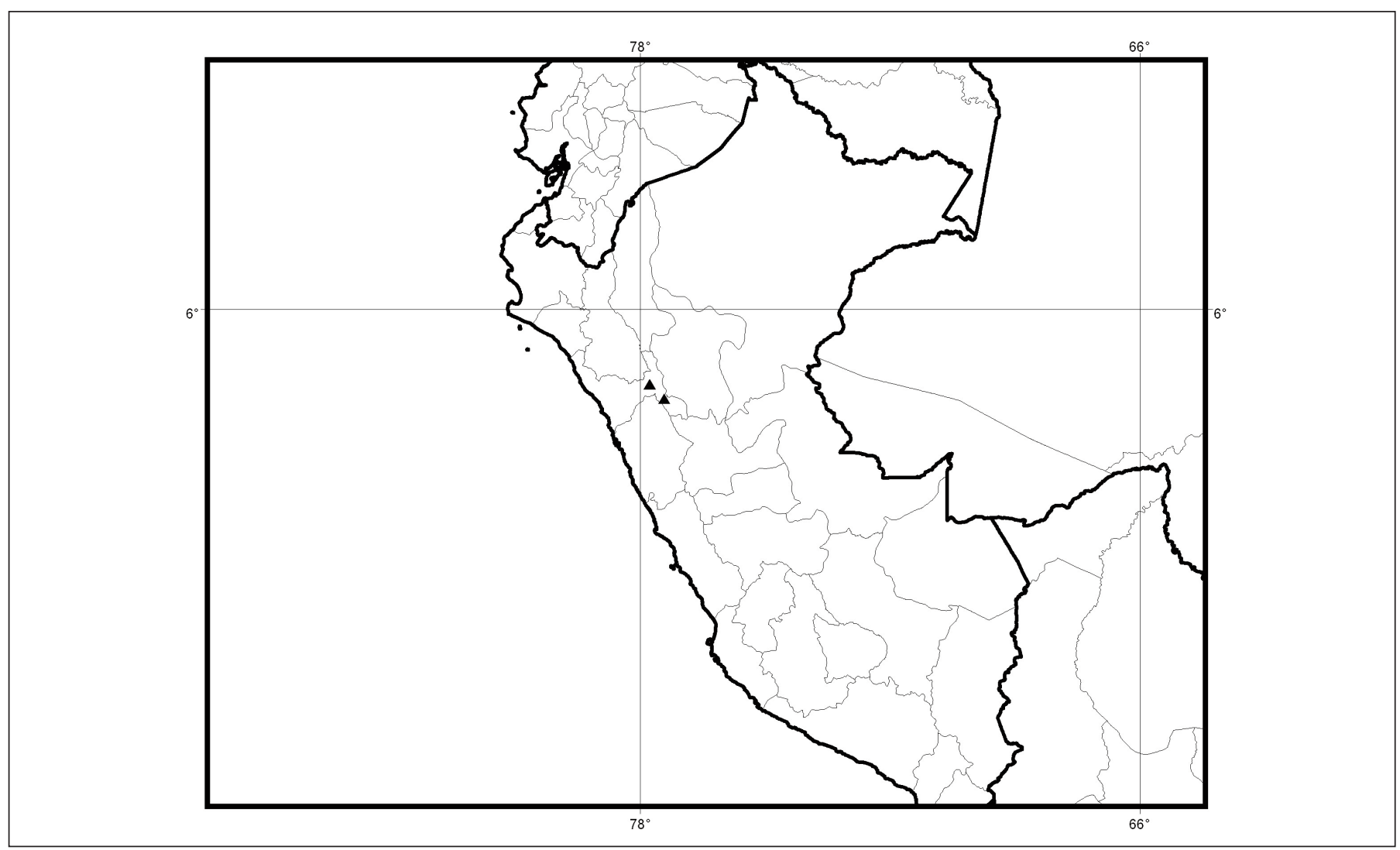

Fig. 2. - Distribución de Geranium trujillense Aedo (

unos $50 \mathrm{~km}$. Solo se ha visitado una de ellas debido a las facilidades de acceso (de Chugay a Molino Viejo). En ella la planta parece muy escasa pues solo se localizaron dos rodales. No obstante hay que señalar las dificultades de localización que se presentan cuando la planta está en fruto debido a su pequeño tamaño y a su mimetización bajo las grandes hierbas de la jalca. No se han detectado amenazas específicas para la conservación de la especie. Cabe señalar únicamente la proximidad de esta localidad a una carretera (unos 100 m). En estas zonas del Perú septentrional las superficies con hábitats similares son muy amplias y es de esperar que prospecciones florísticas detalladas permitan localizar más poblaciones con las que se pueda completar la descripción de la especie.

\section{Clave de las especies seríceas de Geranium sect. Neoandina}

En Aedo $(2004,2007)$ se presentan sucesivas actualizaciones de la clave para identificar las especies de la sect. Neoandina. En esta ocasión se ha preferido omitir las especies de hoja glabra o algo pelosa, y renovar únicamente la parte de la clave que se refiere a las especies de hoja serícea. Se señalan con un asterisco las especies peruanas y con dos aquellas simpátricas con G. trujillense.
1. Hoja serícea por el haz y glabra por el envés G. humboldtii Spreng.

1a. Hoja serícea por ambas caras 2

2. Lámina foliar digitada, con los segmentos laterales rectos o incurvados 3

2a. Lámina foliar palmatífida o palmatisecta, con los segmentos laterales patentes o recurvados.

4

3. Pecíolos con pelos patentes a retrorsos; rostrum con un ápice estrechado de 1,6-2 $\mathrm{mm}$.

G. digitatum

3a. Pecíolos con pelos antrorsos; rostrum sin ápice estrechado G. trujillense

4. Lámina foliar palmatisecta 5

4a. Lámina foliar palmatipartida ................................... 8

5. Pecíolo seríceo, con pelos antrorsos ........................... 6

5a. Pecíolo seríceo, con pelos patentes o retrorsos............ 7

6. Pétalos de 4-7 mm; nectarios glabros; rostrum del fruto sin estrechamiento apical

G. tovarii Aedo

6a. Pétalos de 10,6-15 mm; nectarios pelosos; rostrum del fruto con un estrechamiento apical de 1-1,7 mm.

G. weddellii Briq. 
7. Lámina foliar 0,45-0,84 cm de longitud; pecíolos con pelos retrorsos, \pm adpresos, de 0,2-0,6 $\mathrm{mm}$; sépalos de 4,5-8,2 $\mathrm{mm}$ G. ecuadoriense Hieron.

7a. Lámina foliar 0,9-1,7 cm de longitud; pecíolos con pelos patentes a retrorsos (no adpresos), de $0,8-1,6 \mathrm{~mm}$; sépalos de 4,5-8,2 $\mathrm{mm}$

G. sericeum Spreng.

8. Lámina foliar $0,2-0,9 \mathrm{~cm}$ de longitud; pelos del pedicelo de $0,1-0,4(-0,6) \mathrm{mm}$. 9

8a. Lámina foliar (0,7-)0,9-1,45 cm de longitud; pelos del pedicelo de 0,4-1,5 $\mathrm{mm}$ 10

9. Sépalos sin mucrón; pétalos de 6-7 mm

G. planum Halloy

9a. Sépalos con un mucrón de 0,2-0,5 mm; pétalos de 7 $10,5 \mathrm{~mm}$

G. crassipes A. Gray.

10. Segmento central y generalmente también los laterales con 3 lóbulos; rostrum del fruto sin estrechamiento apical G. ruizii Hieron.

10a. Todos los segmentos enteros; rostrum del fruto con un estrechamiento apical de 1,2-1,5 mm G. nivale R. Knuth

\section{Agradecimientos}

Este trabajo fue financiado con cargo al proyecto de investigación CGL2010-19747 del Ministerio de Ciencia e Innovación. Agradezco la traducción al latín de la diagnosis y las sugerencias sobre el epíteto a M. Laínz. M. Zapata y S. Leiva apoyaron la organización del viaje para recolectar la nueva especie y el primero de ellos tuvo la amabilidad de participar en la misma.

\section{Referencias}

AEdo, C. (2001). Taxonomic revision of Geranium sect. Brasiliensia (Geraniaceae). Syst. Bot. 26: 205-215.

Aedo, C. (2004). A new species of Geranium sect. Neoandina (Geraniaceae) from Peru. Harvard Pap. Bot. 9: 1-4.

AEDo, C. (2007). A new species of Geranium sect. Neoandina (Geraniaceae) from Colombia. J. Torrey Bot. Soc. 134: 534-539.

AEdo, C., J. J. Aldasoro \& C. NAVARro (2002). Revision of Geranium sections Azorelloida, Neoandina, and Paramensia (Geraniaceae). Blumea 47: 205-297.

Aedo, C., J. J. Aldasoro, L. Sáez \& C. Navarro (2003). Taxonomic revision of Geranium sect. Gracilia (Geraniaceae). Brittonia 55: 93-126.

Aedo, C., C. Navarro \& M. L. Alarcón (2005). Taxonomic revision of Geranium sections Andina and Chilensia (Geraniaceae). Bot. J. Linn. Soc. 149: 1-68.

KNUth, R. (1912). Geranium L. In: Engler, A. (ed.), Pflanzenr. 53 : 43-221, 575-583. Leizpig.

Macbride, J. F. (1949). Geranium L. Geraniaceae. Flora of Peru. Publ. Field Mus. Nat. Hist., Bot. Ser. 13: 511-538. 